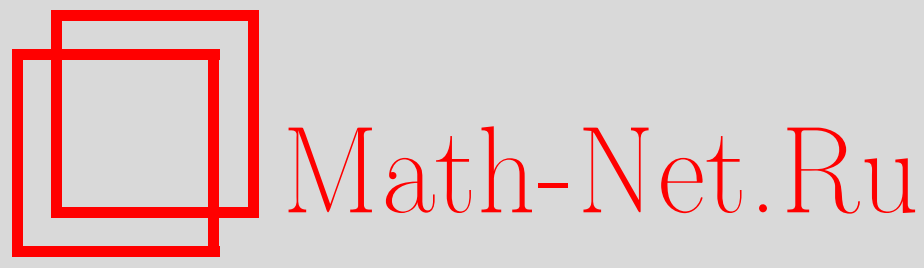

С. Н. Лобанов, Распределение длины и высоты трендов для броуновского движения со сносом, Теория вероятн. и ее примен., 2005, том 50, выпуск 4, 783-789 DOI: https://doi.org/10.4213/tvp134

Использование Общероссийского математического портала Math-Net.Ru подразумевает, что вы прочитали и согласны с пользовательским соглашением

http: //www . mathnet.ru/rus/agreement

Параметры загрузки:

IP : 35.173 .219 .12

26 апреля 2023 г., $11: 44: 32$

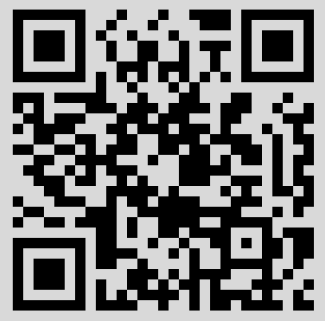




\title{
РАСПРЕДЕЛЕНИЕ ДЛИНЫ И ВЫСОТЫ ТРЕНДОВ ДЛЯ БРОУНОВСКОГО ДВИЖЕНИЯ СО СНОСОМ
}

\begin{abstract}
Понятие тренда является одним из основных при описании поведения какого-либо процесса, развивающегося во времени. Существует по меньшей мере несколько определений трендов, и зачастую при использовании этого понятия даже не уточняется, что конкретно имеется в виду. В данной работе приводятся два наиболее часто используемых определения тренда и устанавливается связь между ними. Также дается описание распределения длины и высоты тренда. В силу особой роли броуновского движения в финансовой математике данные характеристики представляют собой отправную точку, относительно которой следует рассматривать их эмпирические аналоги.
\end{abstract}

Ключевые слова и фразы: тренд, длина тренда, высота тренда, броуновское движение со сносом.

1. Введение. Понятие тренда часто употребляется финансовыми аналитиками при описании поведения рынка или отдельных акций. Несмотря на высокую популярность этого термина, многие аналитики наверняка затруднятся его достаточно четко определить. Неоднозначность определения, что́, собственно говоря, является трендом, легко видеть, например, в статье [4], где исследуется вопрос моделирования трендов в экономике США. Одним из интересных результатов работы [4] является, в частности, следующее эмпирическое наблюдение: сложные модели типа GARCH для квартального процесса ВВП дают лучшее приближение для реального процесса по сравнению с нормальным случайным блужданием. Однако более сложные модели давали менее точное описание трендов, чем простое случайное блуждание.

В связи с вышеизложенным представляется интересным получить распределение высоты и длины тренда для такого классического процесса, как броуновское движение со сносом. В п. 2 данной работы приводятся два определения тренда и устанавливаются их основные свойства. В п. 3 устанавливается совпадение законов распределения пары «длина и высота тренда» для двух приведенных определений, а также выводится преобразование Лапласа для совместного распределения длины и высоты тренда. Здесь же выводятся плотности распределения для длины и высоты тренда, а также получаются условные распределения Лапласа. В п. 4 резюмируются результаты данной работы и обсуждаются дальнейшие направления исследования.

2. Определение тренда и его высоты. Прежде всего определим участки постоянного тренда и моменты смена тренда. Пусть $\mathrm{B}_{t}^{\lambda}=\lambda t+\sigma \mathrm{B}_{t}-$ броуновское движение со сносом. Введем понятие тренда. Для этого определим следующие два момента. Момент первой смены тренда:

$$
\tilde{\tau}_{1}^{d}=\inf \left\{t: \sup _{0 \leqslant s \leqslant t} \mathrm{~B}_{s}^{\lambda}-\mathrm{B}_{t}^{\lambda}=d\right\}
$$

и момент перелома тренда:

$$
\widetilde{\sigma}_{1}^{d}=\inf \left\{0 \leqslant t \leqslant \widetilde{\tau}_{1}^{d}: \sup _{0 \leqslant s \leqslant t} \mathrm{~B}_{s}^{\lambda}=\sup _{0 \leqslant s \leqslant \tilde{\tau}_{1}^{d}} \mathrm{~B}_{s}^{\lambda}\right\} .
$$

Далее рекуррентно определяем момент второй смены тренда (с убываюшего на возрастающий):

$$
\tilde{\tau}_{2}^{d}=\inf \left\{\tilde{\sigma}_{1}^{d} \leqslant t: \mathrm{B}_{t}^{\lambda}-\inf _{\tilde{\sigma}_{1}^{d} \leqslant s \leqslant t} \mathrm{~B}_{s}^{\lambda}=d\right\},
$$

* Математический институт им. В. А. Стеклова РАН, ул. Губкина, 8, 119991 Москва, ГСП-1, Россия; e-mail: me@slobanov.ru 
и момент перелома тренда (с убывающего на возрастающий):

$$
\tilde{\sigma}_{2}^{d}=\inf \left\{\widetilde{\sigma}_{1}^{d} \leqslant t \leqslant \widetilde{\tau}_{2}^{d}: \inf _{\tilde{\sigma}_{1}^{d} \leqslant s \leqslant t} \mathrm{~B}_{s}^{\lambda}=\inf _{\tilde{\sigma}_{1}^{d} \leqslant s \leqslant \tau_{2}^{d}} \mathrm{~B}_{s}^{\lambda}\right\} .
$$

Следующие моменты, соответственно для перехода от возрастающего к убывающему, будут опять определяться как $\widetilde{\tau}_{3}^{d}=\inf \left\{\widetilde{\sigma}_{2}^{d} \leqslant t: \sup _{\tilde{\sigma}_{2}^{d} \leqslant s \leqslant t} \mathrm{~B}_{s}^{\lambda}-\mathrm{B}_{t}^{\lambda}=d\right\}, \widetilde{\sigma}_{3}^{d}=\inf \left\{\widetilde{\sigma}_{2}^{d} \leqslant\right.$ $\left.t \leqslant \widetilde{\tau}_{3}^{d}: \sup _{\tilde{\sigma}_{2}^{d} \leqslant s \leqslant t} \mathrm{~B}_{s}^{\lambda}=\sup _{\bar{\sigma}_{2}^{d} \leqslant s \leqslant \tau_{3}^{d}} \mathrm{~B}_{s}^{\lambda}\right\}$, и т.д. Легко видеть, что эти определения корректны в том плане, что для любого $k>1$ выполнены неравенства $\widetilde{\tau}_{k}^{d}>\widetilde{\tau}_{k-1}^{d}$ и $\widetilde{\sigma}_{k}^{d} \geqslant \widetilde{\tau}_{k-1}^{d}$ почти наверное. Кроме того, для любого $k \geqslant 1 \widetilde{\tau}_{k}^{d}$ является марковским моментом остановки:

$$
\tilde{\tau}_{2}^{d}=\inf \left\{\tilde{\sigma}_{1}^{d} \leqslant t: \mathrm{B}_{t}^{\lambda}-\inf _{\bar{\sigma}_{1}^{d} \leqslant s \leqslant t} \mathrm{~B}_{s}^{\lambda}=d\right\}=\inf \left\{\tilde{\sigma}_{1}^{d} \leqslant t: \mathrm{B}_{t}^{\lambda}-\inf _{\tilde{\tau}_{1}^{d} \leqslant s \leqslant t} \mathrm{~B}_{s}^{\lambda}=d\right\} .
$$

3. Распределение высоты и длины трендов. Основные результаты. Наибольший интерес, прежде всего, представляет распределение следующих характеристик: длины интервала постоянства тренда

$$
\sigma_{k}^{d}=\tilde{\sigma}_{k}^{d}-\widetilde{\sigma}_{k-1}^{d}
$$

и длины отрезка между точками, в которых были обнаружены смены тренда:

$$
\tau_{k}^{d}=\widetilde{\tau}_{k}^{d}-\widetilde{\tau}_{k-1}^{d}
$$

(для нечетных $k$ эти характеристики соответствуют возрастающим трендам, а для четных $k$ - убываюшим). Для упрошения дальнейших выкладок обратим внимание на то, что законы распределения не изменятся, если считать $\sigma=1$ и заменить параметры на $\lambda / \sigma$ и $d / \sigma$. Поэтому при последующем изложении будем считать, что $\sigma=1$. Большой интерес представляет распределение «высоты тренда». Данную характеристику можно определять «предсказуемым» и «не предсказуемым» образом. А именно, как модуль разности процесса в точках обнаружения смены тренда и в точках перелома тренда: $\widetilde{H}_{k}^{d}=\left|\mathrm{B}_{\tilde{\tau}_{k}^{d}}^{\lambda}-\mathrm{B}_{\tilde{\tau}_{k-1}^{d}}^{\lambda}\right|, H_{k}^{d}=\left|\mathrm{B}_{\tilde{\sigma}_{k}^{d}}^{\lambda}-\mathrm{B}_{\tilde{\sigma}_{k-1}^{d}}^{\lambda}\right|$. Легко заметить, что данные способы определения высоты тренда очень тесно связаны, а именно, $H_{k}^{d}=\widetilde{H}_{k}^{d}+2 d$.

Совместное распределение расстояния между точками обнаружения смены тренда и «предсказуемой» высоты тренда для нечетных $k$ впервые было получено в работе [5] и описывается следующим преобразованием Лапласа:

$$
\mathbf{E} \exp \left(-u \widetilde{H}_{k}^{d}-s \tau_{k}^{d}\right)=\frac{\delta \exp (-(\lambda-u) d)}{\delta \operatorname{ch}(\delta d)-(\lambda-u) \operatorname{sh}(\delta d)},
$$

где $\delta=\sqrt{2 s+\lambda^{2}}$. Отсюда, в частности, следует, что

$$
\mathbf{E} \exp \left(-s \tau_{k}^{d}\right)=\frac{\delta \exp (-\lambda d)}{\delta \operatorname{ch}(\delta d)-\lambda \operatorname{sh}(\delta d)}, \quad \mathbf{E} \exp \left(-u \widetilde{H}_{k}^{d}\right)=\frac{\lambda \exp (-(\lambda+u) d)}{\lambda \operatorname{ch}(\lambda d)-(\lambda-u) \operatorname{sh}(\lambda d)} .
$$

Для четных $k$ в приведенных выше формулах потребуется заменить $\lambda$ на $-\lambda$. Соответственно, основной задачей является получение совместного распределения $\sigma_{k}^{d}$ и $H_{k}^{d}$.

Теорема 1. Совместное распределение случайных величин $\sigma_{k}^{d}$ u $H_{k}^{d}$ совпадает с совместным распределением величин $\tau_{k}^{d}$ и $\widetilde{H}_{k}^{d}+2 d$.

Д о к а з а т е л ь с т в о. Для проведения доказательства удобно рассмотреть следуюшие две величины: $\nu_{k}^{d}=\widetilde{\sigma}_{k}^{d}-\widetilde{\tau}_{k-1}^{d}$ и $\varkappa_{k}^{d}=\widetilde{\tau}_{k}^{d}-\widetilde{\sigma}_{k}^{d}$. Фактически это разбиение $\tau_{k}^{d}$ на два отрезка - до момента выхода на максимум (минимум) и после. Рассмотрим нечетные $k$. Для них все моменты фактически определялись относительно процесса $\sup _{0 \leqslant s \leqslant t} \mathrm{~B}_{s}^{\lambda}-\mathrm{B}_{t}^{\lambda}$. Согласно работе [3], данный процесс распределен как модуль «bangbang»-процесса $\left|X_{t}^{\lambda}\right|$, который определяется как единственное решение следуюшего стохастического дифференциального уравнения:

$$
\mathrm{d} X_{t}^{\lambda}=-\lambda \operatorname{sign} X_{t}^{\lambda} \mathrm{d} t+\mathrm{dB}_{t}, \quad X_{0}^{\lambda}=0 .
$$


Мера данного процесса абсолютно локально непрерывна относительно винеровской меры, и ее плотность описывается следуюшей формулой:

$$
\exp \left(-\lambda\left(\left|\mathrm{B}_{t}\right|-\mathrm{L}_{t}\right)-\frac{\lambda^{2} t}{2}\right)
$$

где $\mathrm{L}_{t}$ - локальное время броуновского движения в нуле. Таким образом, получается, что $\tau_{k}^{d}$ будет соответствовать моменту выхода на уровень $d$ процесса $X_{t}^{\lambda}$. Момент $\varkappa_{k}^{d}$ будет соответствовать выходу на уровень $d$ экскурсии, вышедшей из последнего нуля на интервале $\left[0, \tau_{k}^{d}\right]$. Если перейти в формуле замены меры к локальному времени $\tau_{l}$, то получим $\exp \left(\lambda l-\lambda^{2} \tau_{l} / 2\right)$. Отсюда очевидно, что мера в пространстве экскурсий для «bang-bang»-ıіроцесса $n^{\lambda}$ может быть представлена как $\exp \left(-\lambda^{2} R / 2\right) n$, где $R$ - длина экскурсии, а $n$ - мера Ито. Пользуясь всем вышеизложенным, утверждением 3.5 из $[2$, гл. $12, \S 3]$ и тем, что момент выхода на уровень является терминальным, получаем

$$
\begin{aligned}
\mathbf{E} \exp \left(-s \varkappa_{k}^{d}\right) & =\widetilde{\mathbf{E}}\left[\exp \left(-\lambda\left(\left|\mathrm{B}_{\hat{\tau}^{d}} \widehat{\tau}^{d}\right|-\mathrm{L}_{\hat{\tau}^{d}}\right)-\frac{\lambda^{2} \widehat{\tau}^{d}}{2}\right) \exp \left(-s \eta^{d}\right) \mid \mathscr{F}_{\hat{\sigma}_{\hat{\tau}^{d}}}\right] \\
& =4 n\left(\exp \left(-\frac{\lambda^{2} R}{2}\right) \exp \left(-s \widehat{\tau}^{d}\right) \mid R>\widehat{\tau}^{d}\right),
\end{aligned}
$$

где $\widetilde{\mathrm{E}}$ - это математическое ожидание по винеровской мере, $\widehat{\sigma}_{t}$ - последний нуль на отрезке $[0, t], \widehat{\tau}^{d}$ - момент выхода процесса на уровень $d$, а $\eta^{d}=\widehat{\tau}^{d}-\widehat{\sigma}_{\hat{\tau}^{d}}$. $\mathrm{C}$ другой стороны, известно, что условно по высоте экскурсия распадается на два независимых процесса Бесселя размерности 3, бегуших вплоть до выхода на уровень, равный высоте экскурсии. Для процессов Бесселя, в свою очередь, хорошо известны моменты выхода на уровень: $\mathbf{E}_{0}^{\text {Bes }} \exp \left(-s \tau^{d}\right)=d \sqrt{2 s} / \operatorname{sh}(d \sqrt{2 s})$. Кроме того, известно распределение максимума экскурсии: $n(M \in \mathrm{d} m)=\mathrm{d} m / m^{2}$. Таким образом, получаем, что

$$
\begin{aligned}
\mathbf{E} \exp \left(-s \varkappa_{k}^{d}\right) & =\frac{1}{n^{\lambda}\left(R>\widehat{\tau}^{d}\right)} \int_{d}^{\infty} \frac{d \sqrt{2\left(\lambda^{2} / 2+s\right)}}{\operatorname{sh}\left(d \sqrt{2\left(\lambda^{2} / 2+s\right)}\right)} \frac{(m-d)|\lambda|}{\operatorname{sh}((m-d)|\lambda|)} \frac{m|\lambda|}{\operatorname{sh}(m|\lambda|)} \frac{\mathrm{d} m}{m^{2}} \\
& =\frac{d \delta}{\operatorname{sh}(d \delta)} \frac{1}{n^{\lambda}\left(R>\widehat{\tau}^{d}\right)} \int_{d}^{\infty} \frac{(m-d)|\lambda|}{\operatorname{sh}((m-d)|\lambda|)} \frac{m|\lambda|}{\operatorname{sh}(m|\lambda|)} \frac{\mathrm{d} m}{m^{2}}=Q(d, \lambda) \frac{d \delta}{\operatorname{sh}(d \delta)},
\end{aligned}
$$

где

$$
Q(d,-\lambda)=Q(d, \lambda)=\left(\int_{d}^{\infty} \frac{(m-d)|\lambda|}{\operatorname{sh}((m-d)|\lambda|)} \frac{m|\lambda|}{\operatorname{sh}(m|\lambda|)} \frac{\mathrm{d} m}{m^{2}}\right)\left(\int_{d}^{\infty} \frac{\lambda^{2} \mathrm{~d} m}{\operatorname{sh}^{2}(m|\lambda|)}\right)^{-1}
$$

Здесь было произведено вычисление $n^{\lambda}\left(R>\widehat{\tau}^{d}\right)$ :

$$
n^{\lambda}\left(R>\widehat{\tau}^{d}\right)=\int_{d}^{\infty}\left(\frac{m|\lambda|}{\operatorname{sh}(m|\lambda|)}\right)^{2} \frac{\mathrm{d} m}{m^{2}}=\int_{d}^{\infty} \frac{\lambda^{2} \mathrm{~d} m}{\operatorname{sh}^{2}(m|\lambda|)} .
$$

В результате для нечетных $k$ имеем:

$$
\mathbf{E} \exp \left(-s \varkappa_{k}^{d}\right)=Q(d, \lambda) \frac{d \delta}{\operatorname{sh}(d \delta)} .
$$

В силу симметрии $Q(d, \lambda)$ и $\delta$ по $\lambda$ сразу получаем, что $\mathbf{E} \exp \left(-s \varkappa_{k}^{d}\right)=\mathbf{E} \exp \left(-s \varkappa_{k-1}^{d}\right)$, т.е. распределение $\varkappa_{k}^{d}$ не зависит от $k$. Кроме того, $\varkappa_{k}^{d}$ не зависит от совместного распределения $\widetilde{H}_{k}^{d}$ и $\nu_{k}^{d}$. Следовательно,

$$
\sigma_{k}^{d}=\nu_{k}^{d}+\varkappa_{k-1}^{d} \stackrel{\operatorname{law}}{=} \nu_{k}^{d}+\varkappa_{k}^{d}=\tau_{k}^{d} .
$$

Таким образом, пользуясь результатами [5] и тем, что уже было доказано, легко получить утверждение теоремы.

Прямым следствием теоремы 1 является, в частности, следующее преобразование Лапласа:

$$
\mathbf{E} \exp \left(-u H_{k}^{d}-s \sigma_{k}^{d}\right)=\frac{\delta \exp (-(\lambda+u) d)}{\delta \operatorname{ch}(d \delta)-(\lambda-u) \operatorname{sh}(d \delta)}
$$


Следует заметить, что из доказательства теоремы 1 легко получить преобразование Лапласа для момента $\nu_{k}^{d}$. Действительно, как было показано, моменты $\nu_{k}^{d}$ и $\varkappa_{k}^{d}$ независимы. Следовательно, $\mathbf{E} \exp \left(-s \tau_{k}^{d}\right)=\mathbf{E} \exp \left(-s\left(\varkappa_{k}^{d}+\nu_{k}^{d}\right)\right)=$ $\mathbf{E} \exp \left(-s \varkappa_{k}^{d}\right) \mathbf{E} \exp \left(-s \nu_{k}^{d}\right)$. Так как $\mathbf{E} \exp \left(-s \varkappa_{k}^{d}\right)$ известно, то получаем, что

$$
\mathbf{E} \exp \left(-s \nu_{k}^{d}\right)=\frac{\mathbf{E} \exp \left(-s \tau_{k}^{d}\right)}{\mathbf{E} \exp \left(-s \varkappa_{k}^{d}\right)}=\frac{1}{Q(d, \lambda)} \frac{\exp (-\lambda d)}{d(\delta \operatorname{cth}(\delta d)-\lambda)} .
$$

Это выражение уже зависит от знака $\lambda$. Таким образом все «различие» между возрастающими и убывающими трендами фактически заключено на отрезке между обнаружением смены предыдушего тренда на новый и точкой перелома тренда.

Из преобразования Лапласа также можно получить средние значения для $H_{k}^{d}$ и $\sigma_{k}^{d}$. Для этого достаточно продифференцировать преобразование Лапласа по аргументу в точке 0. Проделав довольно утомительные выкладки, можно получить:

$$
\begin{aligned}
\mathbf{E} \sigma_{k}^{d} & =\frac{\exp (-\lambda d)(\lambda d \operatorname{ch}(\lambda d)-(1+\lambda d) \operatorname{sh}(\lambda d))}{\lambda^{2}(\operatorname{ch}(\lambda d)-\operatorname{sh}(\lambda d))^{2}}=4 \frac{d}{\lambda}+2 \frac{\exp (-2 \lambda d)-1}{\lambda^{2} \exp (-2 \lambda d)} \\
\mathbf{E} H_{k}^{d} & =\frac{-\lambda \exp (-\lambda d)(\lambda d \operatorname{ch}(\lambda d)+(1-\lambda d) \operatorname{sh}(\lambda d))}{\lambda^{2}(\operatorname{ch}(\lambda d)-\operatorname{sh}(\lambda d))^{2}}=2 d \frac{\lambda \exp (-2 \lambda d)-1}{\lambda \exp (-2 \lambda d)}+\frac{1-3 \lambda}{\lambda} .
\end{aligned}
$$

Основываясь на приведенной выше замене меры и установленном совпадении по распределению, можно также получить другие характеристики, которые описывают распределения $\sigma_{k}^{d}$ и $H_{k}^{d}$.

Теорема 2. Условные распределения случайных величин $\sigma_{k}^{d} u H_{k}^{d}$ можно охарактеризовать следуюшими преобразованиями Лапласа для четных $k$ :

$$
\begin{aligned}
& \mathbf{E}\left\{\exp \left(-s \sigma_{k}^{d}\right) ; H_{k}^{d}=y\right\}=\exp \left(-\frac{\delta \operatorname{ch}(d \delta)-\lambda \operatorname{sh}(d \delta)}{\operatorname{sh}(d \delta)}(y-d)\right) \frac{\delta \exp (-\lambda d)}{\operatorname{sh}(d \delta)}, \\
& \mathbf{E}\left\{\exp \left(-u H_{k}^{d}\right) ; \sigma_{k}^{d}=t\right\}=\exp \left(-u d-\lambda d-\frac{\lambda^{2} t}{2}\right) \\
& \quad \times \sum_{k=0}^{\infty} \frac{(-1)^{k} k !(u-\lambda)^{k}}{2^{k}} \sum_{l=0}^{k} \frac{(-1)^{l}}{(k-l) ! l !} c_{t}(-k, k+1, d, k d-2 l d),
\end{aligned}
$$

$2 \partial e$

$$
\mathbf{E}(\xi(\omega) ; \eta(\omega) \in \mathrm{d} y)=\frac{\partial}{\partial y} \mathbf{E}(\xi(\omega) ; \eta(\omega)<y) \mathrm{d} y,
$$

$$
\begin{gathered}
c_{y}(\mu, \nu, t, z)=\mathscr{L}_{s}^{-1}\left(\frac{(2 s)^{\mu / 2}}{\operatorname{ch}^{\nu}(t \sqrt{2 s})} \exp (-z \sqrt{2 s})\right) \\
=2^{\nu} \sum_{k=0}^{\infty} \frac{(-1)^{k} \Gamma(\nu+k) \exp \left(-(\nu t+z+2 k t)^{2} / 4 y\right)}{\sqrt{2 \pi} y^{1+\mu / 2} \Gamma(\nu) k !} D_{\mu+1}\left(\frac{\nu t+z+2 k t}{\sqrt{y}}\right), \\
D_{n}=(-1)^{n} \exp \left(-\frac{x^{2}}{4}\right) \frac{\mathrm{d}^{n}}{\mathrm{~d} x^{n}} \exp \left(-\frac{x^{2}}{2}\right)
\end{gathered}
$$

$u \mathscr{L}_{s}^{-1}$ - преобразование Мапласа по переменной $s$.

Кроме того, можно утверждать, что $H_{k}^{d}$ и $\sigma_{k}^{d}$ для четных $k$ имеют следуюшие плотности:

$$
\begin{aligned}
& \mathbf{P}\left\{H_{k}^{d}=y\right\}=\exp \left(-\frac{\lambda \exp (-\lambda d)}{\operatorname{sh}(\lambda d)}(y-d)\right) \frac{\lambda \exp (-\lambda d)}{\operatorname{sh}(\lambda d)}, \\
& \mathbf{P}\left\{\sigma_{k}^{d}=y\right\}=\exp \left(-\lambda d-\frac{\lambda^{2} t}{2}\right) \sum_{k=0}^{\infty} \frac{k ! \lambda^{k}}{2^{k}} \sum_{l=0}^{k} \frac{(-1)^{l}}{(k-l) ! l !} c_{t}(-k, k+1, d, k d-2 l d) .
\end{aligned}
$$

Д о к а $з$ а т е л ь с т в о. Имеем:

$\mathbf{P}\left\{H_{k}^{d} \in \mathrm{d} y, \sigma_{k}^{d} \in \mathrm{d} t\right\}=\mathbf{P}\left\{\widetilde{H}_{k}^{d} \in \mathrm{d}(y-2 d), \tau_{k}^{d} \in \mathrm{d} t\right\}=\mathbf{P}\left\{\overline{\mathrm{B}}_{\hat{\tau}^{d}}^{\lambda} \in \mathrm{d}(y-d), \widehat{\tau}^{d} \in \mathrm{d} t\right\}$, 
где $\widehat{\tau}^{d}=\min \left\{t: \sup _{0 \leqslant s \leqslant t} \mathrm{~B}_{s}^{\lambda}-\mathrm{B}_{t}^{\lambda} \geqslant d\right\}-$ момент выхода процесса $\sup _{0 \leqslant s \leqslant t} \mathrm{~B}_{s}^{\lambda}-\mathrm{B}_{t}^{\lambda}$ на уровень $d$, а $\overline{\mathrm{B}}_{t}^{\lambda}=\sup _{0 \leqslant s \leqslant t} \mathrm{~B}_{s}^{\lambda}$. Согласно доказанному в работе [3], эту цепочку равенств можно продолжить следуюшим образом:

$$
\begin{aligned}
& \mathbf{P}\left\{\overline{\mathrm{B}}_{\hat{\tau}^{d}}^{\lambda} \in \mathrm{d}(y-d), \widehat{\tau}^{d} \in \mathrm{d} t\right\}=\mathbf{P}\left\{\mathrm{L}_{\hat{\tau}^{d}}\left(X^{\lambda}\right) \in \mathrm{d}(y-d), \widehat{\tau}^{d} \in \mathrm{d} t\right\} \\
& \quad=\left.\exp \left\{-\lambda\left(\left|\mathrm{B}_{\hat{\tau}^{d}}\right|-\mathrm{L}_{\hat{\tau}^{d}}\right)-\frac{\lambda^{2} \widehat{\tau}^{d}}{2}\right\}\right|_{\mathrm{B}_{\hat{\tau}^{d}}=\mathrm{d}, \mathrm{L}_{\hat{\tau}^{d}}=y-d, \tau^{d}=t} \mathbf{P}\left\{\mathrm{L}_{\hat{\tau}^{d}} \in \mathrm{d}(y-d), \widehat{\tau}^{d} \in \mathrm{d} t\right\} \\
& \quad=\exp \left(-\lambda(2 d-y)-\frac{\lambda^{2} t}{2}\right) \mathbf{P}\left\{\mathrm{L}_{\hat{\tau}^{d}} \in \mathrm{d}(y-d), \widehat{\tau}^{d} \in \mathrm{d} t\right\},
\end{aligned}
$$

где $X_{t}^{\lambda}$ определяется как единственное решение уравнения

$$
\mathrm{d} X_{t}^{\lambda}=-\lambda \operatorname{sign} X_{t}^{\lambda} \mathrm{d} t+\mathrm{dB}_{t}, \quad X_{0}^{\lambda}=0 .
$$

Момент $\widehat{\tau}^{d}$ в последних двух выражениях в (1) - это уже момент выхода из $(-d, d)$ процессов $\left|X_{t}^{\lambda}\right|$ и $\mathrm{B}_{t}$ соответственно. Последний переход в (1) использует то, что мера, порождаемая процессом $X^{\lambda}$, локально абсолютно непрерывна относительно винеровской меры и ее плотность выглядит как

$$
\exp \left(-\lambda\left(\left|\mathrm{B}_{t}\right|-\mathrm{L}_{t}\right)-\frac{\lambda^{2} t}{2}\right)
$$

Эта замена меры позволяет с легкостью получить многие характеристики совместного распределения. Прежде всего, с помощью этого подхода можно значительно проще, чем в работе [5], получить совместное преобразование Лапласа для длины и высоты тренда. Действительно, согласно формуле 3.3 .3 из [1],

$$
\operatorname{E} \exp \left(-u \mathrm{~L}_{\hat{\tau}^{d}}-s \widehat{\tau}^{d}\right)=\frac{\sqrt{2 s}}{\sqrt{2 s} \operatorname{ch}(d \sqrt{2 s})+u \operatorname{sh}(d \sqrt{2 s})} .
$$

Следовательно, используя приведенную выше замену меры, получаем

$$
\mathbf{E} \exp \left(-u H_{k}^{d}-s \sigma_{k}^{d}\right)=\frac{\delta \exp (-(\lambda+u) d)}{\delta \operatorname{ch}(d \delta)-(\lambda-u) \operatorname{sh}(d \delta)},
$$

где $\delta=\sqrt{2 s+\lambda^{2}}$. Если воспользоваться формулой

$$
\mathrm{E}\left\{\exp \left(-s \widehat{\tau}^{d}\right) ; \mathrm{L}_{\hat{\tau}^{d}}=y\right\}=\exp \left(-\frac{\sqrt{2 s} \operatorname{ch}(d \sqrt{2 s}) y}{\operatorname{sh}(d \sqrt{2 s})}\right) \frac{\sqrt{2 s}}{\operatorname{sh}(d \sqrt{2 s})}
$$

(см. [1, формула 3.3.4]), то с помошью замены меры можно получить, например, следующее условное преобразование Лапласа:

$$
\mathbf{E}\left\{\exp \left(-s \sigma_{k}^{d}\right) ; H_{k}^{d}=y\right\}
$$

Обозначим совместные плотности распределения $\sigma_{k}^{d}, H_{k}^{d}$ и $\widehat{\tau}^{d}, \mathrm{~L}_{\hat{\tau}^{d}}$ как $\mathbf{f}_{\sigma_{k}^{d}, H_{k}^{d}}(t, y)$ и $\mathbf{g}_{\hat{\tau}^{d}, \mathrm{~L}_{\hat{\tau} d}}(t, y)$. Имеем:

$$
\begin{aligned}
\mathbf{E} & \left\{\exp \left(-s \sigma_{k}^{d}\right) ; H_{k}^{d}=y\right\}=\int_{0}^{\infty} \exp (-s t) \mathbf{f}_{\sigma_{k}^{d}, H_{k}^{d}}(t, y) \mathrm{d} t \\
& =\int_{0}^{\infty} \exp (-s t)\left[\exp \left(-\lambda(2 d-y)-\frac{\lambda^{2} t}{2}\right) \mathbf{g}_{\hat{\tau}^{d}, \mathrm{~L}_{\hat{\tau}^{d}}}(t, y-d)\right] \mathrm{d} t \\
& =\exp (-\lambda(2 d-y)) \mathbf{E}\left\{\exp \left(-\left(s+\frac{\lambda^{2}}{2}\right) \widehat{\tau}^{d}\right) ; \mathrm{L}_{\hat{\tau}^{d}}=y-d\right\} \\
& =\exp \left(-\frac{\delta \operatorname{ch}(d \delta)(y-d)}{\operatorname{sh}(d \delta)}-\lambda(d-(y-d))\right) \frac{\delta}{\operatorname{sh}(d \delta)} \\
& =\exp \left(-\frac{\delta \operatorname{ch}(d \delta)-\lambda \operatorname{sh}(d \delta)}{\operatorname{sh}(d \delta)}(y-d)\right) \frac{\delta \exp (-\lambda d)}{\operatorname{sh}(d \delta)} .
\end{aligned}
$$


Отсюда легко заметить, что, положив $s=0$, можно получить плотность распределения $H_{k}^{d}$. При $s=0$ выполнено $\delta=|\lambda|$, но если внимательно проанализировать приведенные выше выражения, то легко заметить, что все модули в них можно раскрыть в силу нечетности функций $x$ и $\operatorname{sh}(x)$. Таким образом, получаем следуюшее выражение для плотности распределения $H_{k}^{d}$ :

$$
\mathbf{f}_{H_{k}^{d}}(y)=\exp \left(-\frac{\lambda \exp (-\lambda d)}{\operatorname{sh}(\lambda d)}(y-d)\right) \frac{\lambda \exp (-\lambda d)}{\operatorname{sh}(\lambda d)},
$$

т.е. $H_{k}^{d}-d$ экспоненциально распределено со средним $\operatorname{sh}(\lambda d) /(\lambda \exp (-\lambda d))$. Теперь, когда известна плотность распределения $H_{k}^{d}$, можно получить непосредственно условное распределение Лапласа для $\sigma_{k}^{d}$, откуда легко выводится условное среднее для $\sigma_{k}^{d}$.

Приведенное выше преобразование Лапласа для пары $H_{k}^{d}, \sigma_{k}^{d}$ легко обрашается по $s$, и таким образом получаем $\mathbf{E}\left\{\exp \left(-u H_{k}^{d}\right) ; \sigma_{k}^{d}=t\right\}$. Действительно, согласно формуле, приведенной в $[1$, с. 615$]$, имеем:

$$
\begin{aligned}
& \mathscr{L}_{s}^{-1}\left(\frac{(2 s)^{\mu / 2} \exp (-x \sqrt{2 s})}{\operatorname{sh}(t \sqrt{2 s})+z \sqrt{2 s} \operatorname{ch}(t \sqrt{2 s})}\right) \\
& =\sum_{k=0}^{\infty} \frac{(-1)^{k} k !}{2^{k} z^{k+1}} \sum_{l=0}^{k} \frac{(-1)^{l}}{(k-l) ! l !} c_{y}(\mu-k-1, k+1, t, x+k t-2 l t) .
\end{aligned}
$$

Теперь, если сравнить (2) с приведенным выше обрашением преобразования Лапласа и вынести $u$ в знаменателе, то, сделав замену

получим

$$
z \rightarrow \frac{1}{u}, \quad \mu \rightarrow 1, \quad t \rightarrow d, \quad x \rightarrow 0, \quad y \rightarrow t
$$

$$
\mathbf{E}\left\{\exp \left(-u \mathrm{~L}_{\hat{\tau}^{d}}\right) ; \widehat{\tau}^{d}=t\right\}=\sum_{k=0}^{\infty} \frac{(-1)^{k} k ! u^{k}}{2^{k}} \sum_{l=0}^{k} \frac{(-1)^{l}}{(k-l) ! l !} c_{t}(-k, k+1, d, k d-2 l d),
$$

а учитывая ту замену меры, которой мы пользовались, легко получить, что

$$
\begin{aligned}
& \mathbf{E}\left\{\exp \left(-u H_{k}^{d}\right) ; \sigma_{k}^{d}=t\right\}=\exp \left(-u d-\lambda d-\frac{\lambda^{2} t}{2}\right) \mathbf{E}\left\{\exp \left(-(u-\lambda) \mathrm{L}_{\hat{\tau}^{d}}\right) ; \widehat{\tau}^{d}=t\right\} \\
& =\exp \left(-u d-\lambda d-\frac{\lambda^{2} t}{2}\right) \\
& \quad \times \sum_{k=0}^{\infty} \frac{(-1)^{k} k !(u-\lambda)^{k}}{2^{k}} \sum_{l=0}^{k} \frac{(-1)^{l}}{(k-l) ! l !} c_{t}(-k, k+1, d, k d-2 l d) .
\end{aligned}
$$

Аналогично тому, как это было сделано для условного по $H_{k}^{d}$ преобразования Лапласа, можно получить плотность распределения $\sigma_{k}^{d}$ :

$$
\mathbf{f}_{\sigma_{k}^{d}}(t)=\exp \left(-\lambda d-\frac{\lambda^{2} t}{2}\right) \sum_{k=0}^{\infty} \frac{k ! \lambda^{k}}{2^{k}} \sum_{l=0}^{k} \frac{(-1)^{l}}{(k-l) ! l !} c_{t}(-k, k+1, d, k d-2 l d) .
$$

Зная яв:тый вид плотности, опять же можно выписать явную формулу для условного преобразования Лапласа и соответственно условного среднего.

4. Заключение. В данной работе установлена связь между двумя наиболее распространенными определениями тренда. Кроме этого, в работе получено совместное преобразование Лапласа для длины и высоты тренда, а также условное преобразование Лапласа длины по высоте тренда и наоборот. Также получена явная формула плотности распределения как для высоты, так и для длины тренда. Основной теоретической задачей, остаюшейся нерешенной на настоящий момент, является получение формулы совместной плотности длины и высоты тренда. Полученные формулы, в особенности формулы для средних, дают некоторую отправную точку, относительно которой следует рассматривать эмпирические результаты по исследованию трендов. 


\section{СПИСОК ЛИТЕРАТУРЫ}

1. Borodin A.N., Salminen P. Handbook of Brownian Motion. Facts and Formulae. Basel: Birkhäuser, 2002, 672 p.

2. Revuz D., Yor M. Continuous Martingales and Brownian Motion. Berlin: SpringerVerlag, 1994, $560 \mathrm{p}$.

3. Graversen S. E., Shiryaev A. N. An extension of P. Lévy's distributional properties to the case of a Brownian motion with drift. - Bernoulli, 2000, v. 6, № 4, p. 615-620.

4. Hess G.D., Iwata S. Measuring and comparing business-cycle features. - J. Bus. Econom. Statist., 1997, v. 15, № 4, p. 432-444.

5. Taylor H. M. A stopped Brownian motion formula. - Ann. Probab., 1975, v. 3, p. 234246.

6. Пастухов С.В. О некоторых вероятностно-статистических методах в техническом анализе. - Теория вероятн. и ее примен., 2004, т. 49, в. 2, с. 297-316.

Поступила в редакцию 16.X.2005

(C) $2005 \Gamma$.

МИЩЕНКО А. С.*

\section{О РАСПРЕДЕЛЕНИИ ВЕРОЯТНОСТЕЙ НЕКОТОРЫХ ФУНКЦИОНАЛОВ ОТ СЛУЧАЙНОГО БЛУЖДАНИЯ}

В теории броуновского движения давно рассматриваются и уже хорошо изучены такие производные от него процессы, как локальное время, время пребывания выше заданного уровня, значение максимума на отрезке, точка достижения этого максимума. Известны одномерные и некоторые совместные распределения этих процессов в фиксированный момент времени, обнаружено множество взаимосвязей между ними.

В работе рассматривается простейшее симметричное случайное блуждание, т.е. блуждание с бернуллиевским шагом, и определяются дискретные аналоги перечисленных выше функционалов. В качестве основного результата доказывается некоторое равенство двух условных трехмерных распределений, связывающее все построенные дискретные случайные величины. Доказательство основывается на довольно интересном преобразовании траекторий случайного блуждания, переставляющем определенным образом положительные и отрицательные экскурсии.

Делается предельный переход к непрерывному времени, с помощью которого устанавливается аналогичное равенство между распределениями функционалов от броуновского движения. Как в случае дискретного, так и в случае непрерывного времени это соотношение ранее нигде не упоминалось.

Ключевые слова и фразы: броуновское движение, случайное блуждание, локальное время, время пребывания, положение максимума, распределение, экскурсии.

* Московский государственный университет им. М.В. Ломоносова, механикоматематический факультет, кафедра теории вероятностей, Ленинские горы, 119992 Москва, Россия; e-mail: andrei.mischenko@mail.ru 